\title{
Mammography casting-type calcification and risk of local recurrence in DCIS: analyses from a randomised study
}

\author{
L Holmberg ${ }^{\star}, 1,2$, Y N S Wong ${ }^{1}$, L Tabár ${ }^{3,4}$, A Ringberg ${ }^{5}$, P Karlsson ${ }^{6}$, L-G Arnesson ${ }^{7}$, K Sandelin ${ }^{8}$, H Anderson $^{9}$, \\ $\mathrm{H}$ Garmo ${ }^{1,2}$, and S Emdin ${ }^{10}$ on behalf of the Swedish Breast Cancer Group \\ ${ }^{1}$ King's College London, Medical School, Division of Cancer Studies, London SE1 9RT, UK; ${ }^{2}$ Regional Cancer Centre, Uppsala \\ University Hospital, Uppsala S-751 85, Sweden; ${ }^{3}$ School of Medicine, University of Uppsala, Uppsala S-791 82, Sweden; \\ ${ }^{4}$ Department of Mammography, Falun Central Hospital, Falun, Sweden; ${ }^{5}$ Department of Plastic and Reconstructive Surgery, \\ University Hospital MAS, Malmö S-205 02, Sweden; ${ }^{6}$ Department of Oncology, Sahlgrenska University Hospital, Gothenburg \\ S-413 45, Sweden; ${ }^{7}$ Department of Surgery, University Hospital, Linköping S-581 85, Sweden; ${ }^{8}$ Department of Surgery, Karolinska \\ University Hospital, Stockholm S-171 76, Sweden; ${ }^{9}$ Department of Cancer Epidemiology, University of Lund, Lund S-221 85, \\ Sweden and ${ }^{10}$ Department of Surgery, Umeå University Hospital, Umeå S-901 85, Sweden
}

Background: We studied the association between mammographic calcifications and local recurrence in the ipsilateral breast.

Methods: Case-cohort study within a randomised trial of radiotherapy in breast conservation for ductal cancer in situ of the breast (SweDCIS). We studied mammograms from cases with an ipsilateral breast event (IBE) and from a subcohort randomly sampled at baseline. Lesions were classified as a density without calcifications, architectural distortion, powdery, crushed stone-like or castingtype calcifications.

Results: Calcifications representing necrosis were found predominantly in younger women. Women with crushed stone or castingtype microcalcifications had higher histopathological grade and more extensive disease. The relative risk (RR) of a new IBE comparing those with casting-type calcifications to those without calcifications was 2.10 (95\% confidence interval (Cl) 0.92-4.80). This risk was confined to in situ recurrences; the RR of an IBE associated with casting-type calcifications on the mammogram adjusted for age and disease extent was 16.4 (95\% Cl 2.20-140).

Conclusion: Mammographic appearance of ductal carcinoma in situ of the breast is prognostic for the risk of an in situ IBE and may also be an indicator of responsiveness to RT in younger women.

A substantial fraction ( $\sim 20 \%)$ of mammography-detected breast lesions are ductal carcinoma in situ (DCIS). As the mammographic appearance of a breast tumour reflects pathological changes of breast anatomy, the biological aggressiveness of a cancer or its precursors is potentially predicted by mammographic characteristics. An association between mammographic findings and histology, especially when microcalcifications are present has been observed (Lenya, 1988; Tabár et al, 2005; Hermann et al, 2009) and the mammographic traits of various tumour types have been linked to the nature of the underlying pathological changes (Hilleren et al, 1991; Goodman et al, 1995; Wilson et al, 1995). Mammographic characteristics have been proposed as a prognostic indicator of breast cancer (Thurfjell et al, 2001; Tabár et al, 2000a, b; Zunzunegui et al, 2003; Tabár et al, 2004). A higher risk of breast cancer death has been shown in the presence of casting-type calcifications as compared with cancers not associated with this mammographic abnormality (Malik et al, 2000; Tabár et al, 2000b; Zunzunegui et al, 2003; Tabár et al, 2004; Pálka et al, 2007).

*Correspondence: Professor L Holmberg; E-mail: Lars.holmberg@kcl.ac.uk

Received 4 July 2012; revised 4 January 2013; accepted 7 January 2013; published online 31 January 2013

(c) 2013 Cancer Research UK. All rights reserved 0007-0920/13 
However, the long-term clinical results predominantly derive from studies on invasive carcinomas.

We report the results from a case-cohort study nested within the national Swedish randomised trial of breast conservation in DCIS, SweDCIS (Holmberg et al, 2008), in which 1046 women were randomly assigned to radiotherapy or control only, following a sector resection for DCIS between 1987 and 1999. We studied the association between the mammographic appearances of microcalcifications, in particular, the casting-type calcification and the risk of local recurrence in the ipsilateral breast and the relationships between the presence of casting-type calcifications and other tumour characteristics.

\section{SUBJECTS AND METHODS}

Enrolment, randomisation and interventions in SweDCIS. The design and main results of SweDCIS have been described earlier (Holmberg et al, 2008). In short, to be eligible for SweDCIS, women had to have undergone breast-conserving surgery for histologically proven DCIS occupying a quadrant or less of the breast and with a clinically negative examination of the axilla. Exclusion criteria included Paget's disease of the nipple, invasive carcinoma or intracystic carcinoma in situ, on-going pregnancy, history of previous or concurrent malignancy (except basal cell carcinoma and treated carcinoma in situ of the uterine cervix). After full informed consent, the women were randomly assigned to $\mathrm{RT}$ or to control. The trial was nationwide, and randomisation was stratified for health care region. The treating physician reported baseline data. The regional ethics committees approved the study.

After a sector resection of the breast, the women were randomised to postoperative radiotherapy of the breast or control only. A macroscopic surgical margin of $1 \mathrm{~cm}$ to the sides was aimed for. The removed skin overlying the lesion and/or Scarpas' fascia constituted the ventral border and the pectoral fascia, the dorsal border. Microscopically free margins were not requested but achieved in $80 \%$ of all removed surgical specimens $(11 \%$ had positive margins and $8.5 \%$ unknown margins) as reported in the clinical pathology reports and on the case record form of the study. The specification dose of radiotherapy was $50 \mathrm{~Gy}$, given in 25 fractions over 5 weeks or $54 \mathrm{~Gy}$ given in two series with a gap of 2 weeks. No woman was lost to follow-up.

Study design. The present study builds on the patients included in a case-cohort study of histopathological risk factors for ipsilateral breast events (IBE) (Ringberg et al, 2007), with the addition of cases with IBE occurring up to the end of 31 July 2005. Women eligible as cases had an IBE of DCIS or invasive cancer that was identified through a full monitoring of all original medical records through the 31 July 2005. Women eligible as a sample from the cohort - henceforth called subcohort - were all women included in the study. Selection of the subcohort was done from the baseline data at inclusion irrespective of any future IBE. For each of the participating six Health Care Regions, the selection was done by day of birth in the month, with different days randomly allocated to the patients from each region. We aimed to sample at least $20 \%$ of the cohort. Three dedicated breast pathologists examined the microscopic slides of the subcohort and all cases included in the study by Ringberg et al (2007). Cases of IBE occurring between 2002 and 31 July 2005 were not histopathologically re-evaluated. The study set-up is illustrated in the flowchart in Figure 1.

Follow-up and clinical events. Patients were initially followed by biannual clinical examination and yearly mammograms for five years and thereafter by clinical examination and mammography on a yearly basis. All medical records were monitored. Furthermore, we searched all inpatient, mammography and pathologic laboratory registers for indications of any IBE in the cohort.

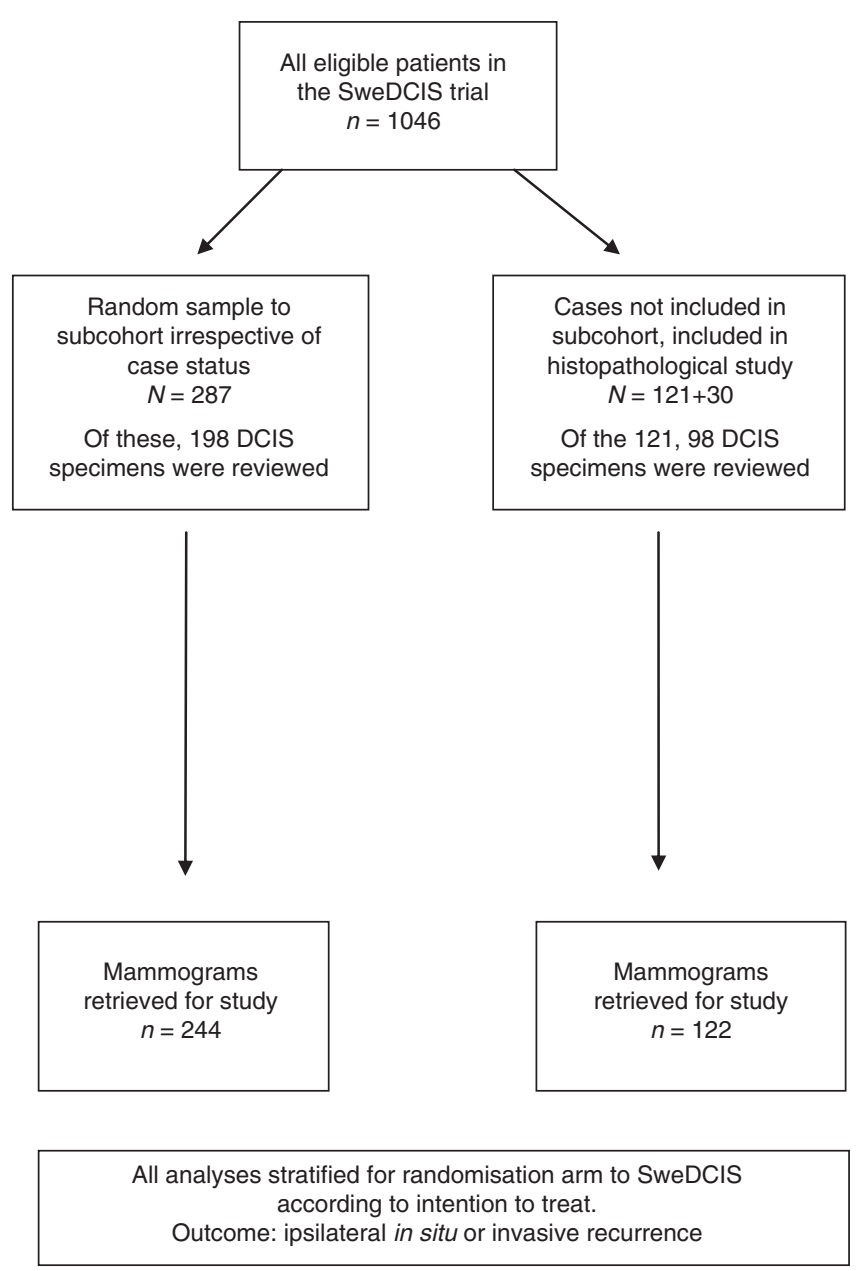

Figure 1. Flow chart of the selection of women to the subcohort and the case-series with ipsilateral breast events (IBE).

All events in the ipsi- or contralateral breast or in the ipsilateral axilla were based on either clinical or mammography diagnosis subsequently morphologically verified after surgical excision. Causes of death were extracted from the Swedish Cause of Death Registry.

Mammographic re-evaluation. We attempted to retrieve all mammographic films from the preoperative evaluation. One experienced radiologist (LT) evaluated the preoperative work-up mammograms and microfocus magnification images, when available. He was blinded to randomisation arm and all clinical data including case status.

The classification of the mammographic findings was based on a description of the mammographic appearance of in situ carcinoma subtypes (Tabár et al, 2005). DCIS cases with no mammographically demonstrable calcifications are called 'special forms' and are detected either by finding an asymmetric density with architectural distortion or a dominant mass (intracystic papillary lesion) or through galactographic findings. Approximately $75-80 \%$ of the mammographically demonstrable in situ breast carcinomas have one or more of the following malignant type calcifications: (1) The crushed stone-like (pleomorphic) calcifications are the most frequently occurring; (2) The 'powdery', cotton ball-like calcifications and (3) The casting -ype, linear, branching calcifications appear in similar proportions.

Statistical methods. Cox proportional hazards models, stratified and adjusted according to specifications in Table 3, were assumed for DCIS and invasive ipsilateral events, and hazard ratios and 
Table 1. Clinical, mammographic and histopathological characteristics of all study subjects. Numbers in each subset vary depending on availability of slides and mammograms

\begin{tabular}{|c|c|c|c|c|}
\hline \multirow[b]{2}{*}{ Age, mean (s.d.) } & \multicolumn{2}{|c|}{ Subcohort } & \multicolumn{2}{|c|}{$\begin{array}{l}\text { Cases outside } \\
\text { subcohort }\end{array}$} \\
\hline & 56.7 & $(9.2)$ & 55.4 & (9.4) \\
\hline \multicolumn{5}{|c|}{ Randomized to, No. (\%) } \\
\hline RT & 148 & $(51.6)$ & 44 & (29.1) \\
\hline Control & 139 & (48.4) & 107 & (70.9) \\
\hline \multicolumn{5}{|c|}{ Detection mode, No. (\%) } \\
\hline Screening detected & 220 & (76.9) & 108 & (71.5) \\
\hline Not screening detected & 66 & (23.1) & 43 & (28.5) \\
\hline \multicolumn{5}{|l|}{ Palpation, No. (\%) } \\
\hline Palpable & 73 & (25.4) & 38 & $(25.2)$ \\
\hline Not palpable & 208 & (72.5) & 109 & (72.2) \\
\hline Unknown & 6 & $(2.1)$ & 4 & $(2.6)$ \\
\hline
\end{tabular}

Tumour size, No. (\%)

\begin{tabular}{|l|c|c|c|c|}
$<10$ & 64 & $(22.3)$ & 23 & $(15.2)$ \\
$10-19$ & 111 & $(38.7)$ & 42 & $(27.8)$ \\
$20-39$ & 70 & $(24.4)$ & 43 & $(28.5)$ \\
$40+$ & 18 & $(6.3)$ & 16 & $(10.6)$ \\
Missing data & 24 & $(8.4)$ & 27 & $(17.9)$ \\
\hline
\end{tabular}

Surgical margins, No. (\%)

\begin{tabular}{l|c|c|c|c|} 
Pathological margins clear & 199 & $(81.6)$ & 86 & $(70.5)$ \\
Pathological margins positive & 26 & $(10.7)$ & 25 & $(20.5)$ \\
$\begin{array}{l}\text { Pathological margins unknown } \\
\text { or missing }\end{array}$ & 19 & $(7.8)$ & 11 & $(9.0)$ \\
\hline
\end{tabular}

Mammographic pattern, No. (\%)

\begin{tabular}{|l|c|c|c|c|}
\hline No calcifications & 32 & $(13.1)$ & 9 & $(7.4)$ \\
Architectural distortion/ & 22 & $(9.0)$ & 8 & $(6.6)$ \\
galactography & & & & \\
Powdery & 25 & $(10.2)$ & 7 & $(5.7)$ \\
Crushed stone-like & 126 & $(51.6)$ & 68 & $(55.7)$ \\
Casting-type & 39 & $(16.0)$ & 30 & $(24.6)$ \\
\hline
\end{tabular}

Cancer type ${ }^{a}$, No. (\%)

Paget/microinvasive/invasive

Benign/ADH/LCIS

Missing/bad material

DCIS

Necrosis among DCIS-patients ${ }^{\mathrm{b}}$, No. (\%)

\begin{tabular}{|l|c|c|c|c|}
\hline Necrosis & 169 & $(73.8)$ & 85 & $(86.7)$ \\
No necrosis & 60 & $(26.2)$ & 13 & (13.3) \\
\hline
\end{tabular}

Nuclear grade among DCIS patients ${ }^{b}$, No. (\%)

\begin{tabular}{|l|c|c|c|c|}
\hline $\mathrm{NG}=1$ & 20 & $(8.7)$ & 3 & $(3.1)$ \\
$\mathrm{NG}=2$ & 95 & $(41.5)$ & 32 & $(32.7)$ \\
$\mathrm{NG}=3$ & 114 & $(49.8)$ & 63 & $(64.3)$ \\
\hline
\end{tabular}

a Only determined for patients included in the pathology study.

bonly determined for patients included in the pathology study with a pathologically verified DCIS

variance estimates for the case-cohort design were determined according to Self and Prentice, (1988). Technically, this was done by using the statistical programme package $\mathrm{R}$ ( $\mathrm{R}$ Development Core Team, 2005) and following Therneau and Li, (1998). The analyses of prognostic factors were stratified on randomisation arm (radiotherapy $v s$ no radiotherapy) to allow for different baseline hazards in the control and RT groups.

The cumulative incidence was determined according to Kalbfleisch and Prentice, (2002) adapted to fit the case-cohort design (the subcohort as previously described under 'Study design') rather than the cohort design, by replacing the numbers at risk by estimated numbers at risk.

In all our time-to-event analyses, follow-up was censored at the end of the study or death if no recurrence had occurred previously. When analysing DCIS events only, follow-up was also censored if a woman first experienced an invasive recurrence. In analyses of invasive events only, follow-up was censored if a woman first had a DCIS recurrence. In estimation of cumulative incidence, death without recurrence was always considered as a competing event to IBE, and when estimating the cumulative incidence of DCIS as first recurrence, an invasive recurrence was also considered as a competing event, and vice versa when estimating the cumulative incidence of an invasive recurrence as first event.

\section{RESULTS}

Table 1 displays the clinical, tumour and mammographic characteristics of the patients included in the subcohort and in the case-series. The numbers in each subset vary according to availability of histopathological data and of mammograms. As expected, the women in the case series had a somewhat lower age at diagnosis, lower proportion screen-detected lesions, larger tumours, not completely excised DCIS, higher proportion of invasive lesions at review, more necrosis and a distribution towards higher grades. In addition, the women in the case series more often had a casting-type mammographic pattern.

We analysed the associations between mammographic pattern and histopathological characteristics in the subcohort population with DCIS as per our previous review (Ringberg et al, 2007) (Figure 1, Table 2). As the subcohort is a random sample of the whole SweDCIS trial, this analysis represents these associations in the underlying study base regardless of case status. When calcifications of any type were present, necrosis was more prevalent. Age seemed to be a determinant for these features as they were more frequently found in younger women. Lesions in women with crushed stone or casting-type microcalcifications also showed higher tumour grade, had a larger diameter and more often had involved margins after surgery (Table 2).

Table 3 shows the relative risks (RRs) with 95\% confidence intervals (CIs) obtained in Cox models of risk of ipsilateral in situ or invasive breast recurrence. All models are stratified by randomisation arm (radiotherapy $v s$ no radiotherapy) in SweDCIS and take the largest patient group - women with crushed stone-like calcifications - as a reference. For all types of IBEs, casting-type and crushed stone-like calcifications implied elevated risks in the non-adjusted model. The RR comparing women with no calcifications to those with casting-type was 2.10 (the ratio of RR: s 1.36/0.65 in Table 3, with 95\% CI 0.92 - 4.8), but with a modest $36 \%$ relative difference in risk between crushed stone and casting-type calcifications.

When we studied in situ and invasive recurrences separately (Table 3), the risk was isolated to the risk of in situ recurrence. In the non-adjusted model, women with casting-type calcifications had a 22.8-times higher risk (1.98/0.09, Table 3, with 95\% CI 2.93-177.21) than those without a calcification to develop an in situ recurrence. Adjustment for age, lesion size and margin status changed the pattern modestly, but the RR for ipsilateral in situ associated with a casting type-was still 16.4 (95\% CI 2.2-140) compared with women without calcifications (Table 3).

Graphs of cumulative incidence of IBEs, by randomisation arm and mammographic patterns characterised as casting-type, crushed 

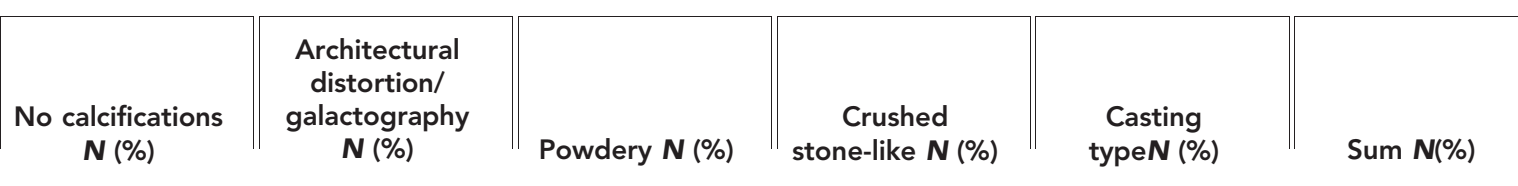

\begin{tabular}{|c|c|c|c|c|c|c|c|c|c|c|c|c|}
\hline $\begin{array}{l}\text { All DCIS in } \\
\text { subcohort }\end{array}$ & 22 & (11.1) & 14 & (7.1) & 20 & $(10.1)$ & 106 & $(53.5)$ & 36 & $(18.2)$ & 198 & (100.0) \\
\hline Necrosis & 9 & (6.1) & 5 & (3.4) & 14 & (9.5) & 86 & (58.5) & 33 & (22.4) & 147 & $(100.0)$ \\
\hline No necrosis & 13 & (25.5) & 9 & (17.6) & 6 & (11.8) & 20 & $(39.2)$ & 3 & (5.9) & 51 & (100.0) \\
\hline $\mathrm{NG}=1$ & 4 & $(25.0)$ & 2 & (12.5) & 2 & (12.5) & 8 & $(50.0)$ & 0 & $(0.0)$ & 16 & $(100.0)$ \\
\hline$N G=2$ & 16 & (19.3) & 7 & (8.4) & 14 & (16.9) & 43 & (51.8) & 3 & (3.6) & 83 & (100.0) \\
\hline$N G=3$ & 2 & (2.0) & 5 & (5.1) & 4 & (4.0) & 55 & $(55.6)$ & 33 & (33.3) & 99 & (100.0) \\
\hline Age $<50$ & 4 & (10.8) & 2 & (5.4) & 7 & (18.9) & 20 & $(54.1)$ & 4 & $(10.8)$ & 37 & (100.0) \\
\hline Age 50-57 & 4 & (6.0) & 1 & (1.5) & 9 & (13.4) & 41 & (61.2) & 12 & (17.9) & 67 & (100.0) \\
\hline Age 58-64 & 3 & (7.1) & 3 & (7.1) & 1 & (2.4) & 24 & (57.1) & 11 & $(26.2)$ & 42 & (100.0) \\
\hline Age $65+$ & 11 & $(21.2)$ & 8 & (15.4) & 3 & (5.8) & 21 & $(40.4)$ & 9 & (17.3) & 52 & $(100.0)$ \\
\hline $\begin{array}{l}\text { Tumour size } \\
<15 \mathrm{~mm}\end{array}$ & 15 & (15.0) & 7 & (7.0) & 9 & (9.0) & 57 & $(57.0)$ & 12 & (12.0) & 100 & (100.0) \\
\hline $\begin{array}{l}\text { Tumour size } \\
15 \geqslant \mathrm{~mm}\end{array}$ & 7 & (8.4) & 5 & (6.0) & 7 & (8.4) & 42 & $(50.6)$ & 22 & $(26.5)$ & 83 & $(100.0)$ \\
\hline $\begin{array}{l}\text { Missing tumour } \\
\text { size }\end{array}$ & 0 & $(0.0)$ & 2 & (13.3) & 4 & $(26.7)$ & 7 & $(46.7)$ & 2 & (13.3) & 15 & $(100.0)$ \\
\hline $\begin{array}{l}\text { Surgical margins } \\
\text { clear }\end{array}$ & 17 & (10.4) & 12 & (7.3) & 18 & (11.0) & 93 & $(56.7)$ & 24 & $(14.6)$ & 164 & (100.0) \\
\hline $\begin{array}{l}\text { Surgical margins } \\
\text { positive }\end{array}$ & 3 & (15.8) & 0 & (0.0) & 2 & (10.5) & 9 & (47.4) & 5 & $(26.3)$ & 19 & $(100.0)$ \\
\hline $\begin{array}{l}\text { Unknown/ } \\
\text { missing }\end{array}$ & 2 & (13.3) & 2 & (13.3) & 0 & $(0.0)$ & 4 & $(26.7)$ & 7 & $(46.7)$ & 15 & $(100.0)$ \\
\hline
\end{tabular}

stone and others grouped together (Figure 2) show again a higher risk associated with crushed stone or casting calcifications (Figure 2, upper panels). The high risk for women with castingtype calcifications pertained mainly to in situ local recurrences (lefthand panel, lower panels Figure 2). The pattern was similar for crushed stone-like calcifications.

Although radiotherapy in relative terms had similar effects in all subsets (Figure 2), in absolute terms, the risk of recurrence was still high in women with casting-type calcifications despite postoperative radiotherapy. The cumulative incidence graphs of in situ local recurrence indicates that the recurrences present at a steady rate during the first four years of follow-up after diagnosis in the casting calcification group, irrespective of whether radiotherapy was given or not (lower left panel, Figure 2). However, the cumulative incidence graphs for invasive recurrence indicates that the rate of invasive recurrences is stable over time at least during the first 8 years of follow up (middle panels, Figure 2).

When the cumulative incidence per randomisation arm and by mammographic pattern was analysed by the median age (56 years at diagnosis) in the study, it appeared that in women $\leqslant 56$ years of age, radiotherapy had less impact on local recurrence than in women $>56$ years of age, especially when casting-type calcifications were present on the mammogram (Figures 3 and 4). In younger women with casting-type calcifications, the cumulative incidence curve for those randomized to radiotherapy even tended to be above those allocated to control (left hand panels Figure 3), but there was no statistically significant interaction between age as a continuous variable and radiotherapy for all $\operatorname{IBE}(P=0.69)$ or for in situ IBE $(P=0.80)$. On the other hand, the high risk for women over 56 years of age with casting calcifications was strongly reduced in the study arm randomized to RT (left hand panels Figure 4).
In the study as a whole, only 14 patients died from breast cancer with no statistically significant difference between the randomisation arms or between the mammographic pattern groups.

\section{DISCUSSION}

We found an increased risk of IBE in patients with crushed stone and casting-type calcifications. Both types of calcifications were associated with a marked predominance with recurrence of ipsilateral in situ carcinomas. The findings - notably from a randomized study design - also indicate that radiotherapy had low efficacy in women with casting-type calcifications and $\leqslant 56$ years at diagnosis, but clearly reduced risk in women over 56 years. In women with casting-type calcifications on the mammogram, in situ recurrences presented at a high and constant rate during the first 4 years of follow-up. In contrast, invasive recurrences had a more constant occurrence rate over the 8 years of observation. As expected, there was a strong association between casting-type calcifications and necrosis as well as histological nuclear grade 3, but the risk estimates derived from the mammographic pattern did not disappear with a correction for clinical or histopathological variables.

The analyses are based on a large randomized trial with long and complete follow-up. The case-cohort study design exhibits a statistical power close to that of analysing the full trial as it uses all events of interest. As the subcohort is a random sample of the entire trial, the randomized design is not compromised. The majority of the mammograms from the subcohorts and cases outside the subcohort were obtained and re-evaluated, and only one experienced radiologist reviewed the mammograms. As we 
Table 3. Relative risks and $95 \% \mathrm{Cls}$ for ipsilateral recurrence obtained in Cox Proportional Hazards Models. All models are stratified for randomisation arm in SweDCIS. 'Other' category denotes architectural distortion and galactography findings

\begin{tabular}{|c|c|c|c|c|c|c|}
\hline \multirow[b]{2}{*}{ Crushed stone-like calcification $^{a}$} & \multicolumn{2}{|c|}{ All ipsilateral } & \multicolumn{2}{|c|}{ Ipsilateral DCIS } & \multicolumn{2}{|c|}{ Ipsilateral Invasive } \\
\hline & Ref & & Ref & & Ref & \\
\hline Casting-type calcification ${ }^{a}$ & 1.36 & $(0.79-2.35)$ & 1.98 & $(1.08-3.65)$ & 0.69 & $(0.30-1.62)$ \\
\hline Powdery calcification ${ }^{\text {a }}$ & 0.54 & $(0.24-1.24)$ & 0.59 & $(0.19-1.80)$ & 0.49 & $(0.17-1.40)$ \\
\hline No calcification ${ }^{a}$ & 0.65 & $(0.31-1.36)$ & 0.09 & $(0.01-0.66)$ & 1.30 & $(0.58-2.91)$ \\
\hline Other ${ }^{a}$ & 0.62 & $(0.27-1.43)$ & 0.34 & $(0.09-1.35)$ & 0.96 & $(0.37-2.53)$ \\
\hline Crushed stone-like calcification ${ }^{\mathbf{b}}$ & Ref & & Ref & & Ref & \\
\hline Casting-type calcification ${ }^{\mathbf{b}}$ & 1.41 & $(0.81-2.45)$ & 2.03 & $(1.09-3.76)$ & 0.72 & $(0.31-1.71)$ \\
\hline Powdery calcification ${ }^{\mathbf{b}}$ & 0.48 & $(0.21-1.11)$ & 0.54 & $(0.18-1.64)$ & 0.43 & $(0.15-1.22)$ \\
\hline No calcification ${ }^{\mathbf{b}}$ & 0.67 & $(0.32-1.41)$ & 0.09 & $(0.01-0.68)$ & 1.35 & $(0.60-3.05)$ \\
\hline Other ${ }^{b}$ & 0.60 & $(0.26-1.40)$ & 0.33 & $(0.08-1.32)$ & 0.93 & $(0.35-2.48)$ \\
\hline Crushed stone-like calcification $^{c}$ & Ref & & Ref & & Ref & \\
\hline Casting-type calcification ${ }^{c}$ & 1.05 & ( 0.56-1.95) & 1.31 & $(0.64-2.66)$ & 0.61 & $(0.24-1.53)$ \\
\hline Powdery calcification ${ }^{c}$ & 0.31 & $(0.12-0.82)$ & 0.31 & (0.09-1.14) & 0.30 & $(0.10-0.94)$ \\
\hline No calcification ${ }^{c}$ & 0.60 & $(0.28-1.31)$ & 0.08 & $(0.01-0.61)$ & 1.23 & $(0.54-2.84)$ \\
\hline Other ${ }^{c}$ & 0.35 & $(0.12-0.99)$ & 0.15 & $(0.03-0.77)$ & 0.67 & $(0.21-2.09)$ \\
\hline \multicolumn{7}{|c|}{$\begin{array}{l}{ }^{a} \text { Stratified on treatment (RT/control). } \\
{ }^{b} \text { Adjusted for age (continuous) and stratified on treatment. } \\
{ }^{c} \text { Adjusted for age (continuous), tumour size (grouped }<15 n\end{array}$} \\
\hline
\end{tabular}
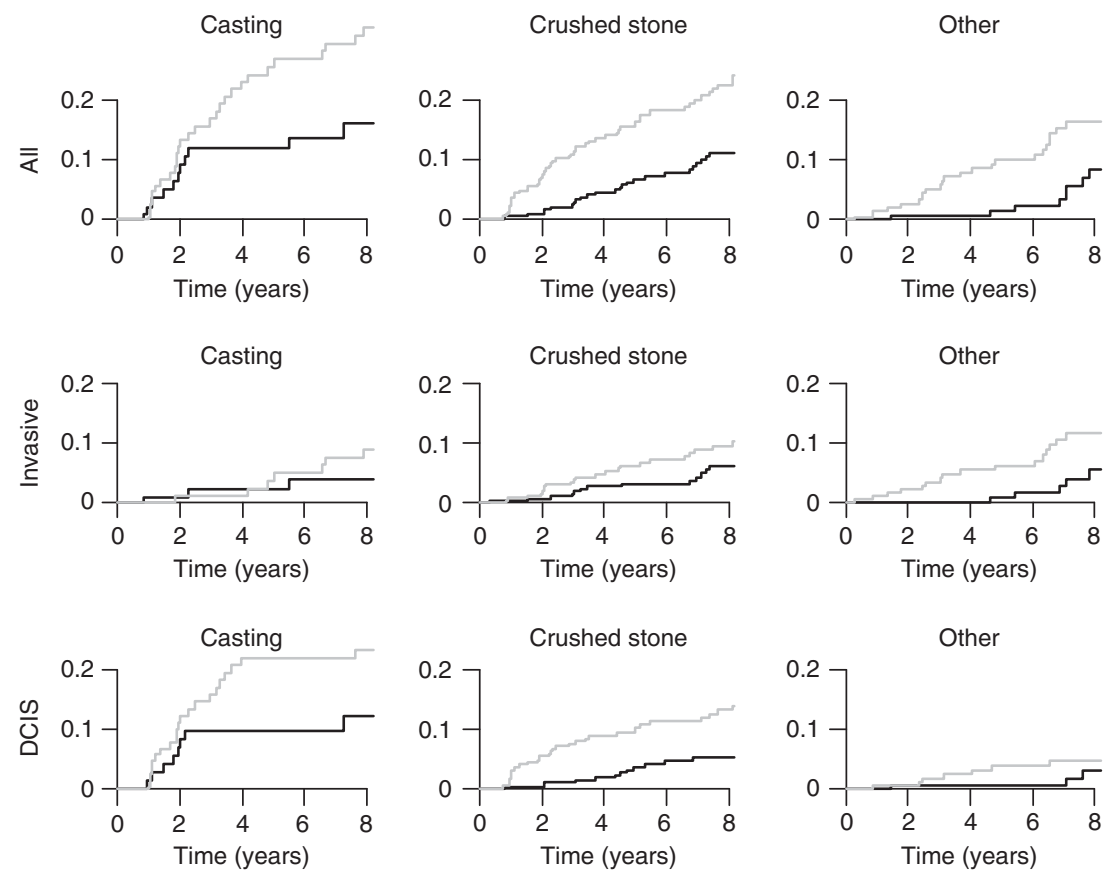

- RT Control

Figure 2. Cumulative incidence of local recurrences by mammographic pattern in all women; the three upper panels describe all (invasive plus in situ) recurrences in the radiotherapy (RT) arm and the control (Ctrl) arm by type of mammographic pattern of the primary lesion; the three middle panels describe invasive recurrences in the radiotherapy (RT) arm and the control (Ctrl) arm, the lower panels the in situ recurrences in the respective arm of the SweDCIS trial by mammographic pattern of the primary. Women with no calcifications, and those with architectural distortion, galactography findings, and with powdery calcifications are grouped together as 'Other'.

could not locate all mammograms and despite that the number of cases is comparatively large, there were a limited number of cases in the subgroups obtained by stratification, which entails a limited statistical power and precision in the subgroup analyses. We also had data from a previous review and re-evaluation of the participants' histopathologal slides done by three experienced breast pathologists (Ringberg et al, 2007). There may have been a misclassification of the primary lesion for some of the cases added after 2001, that is some four per cent may have had an invasive tumour (Emdin et al, 2006). 

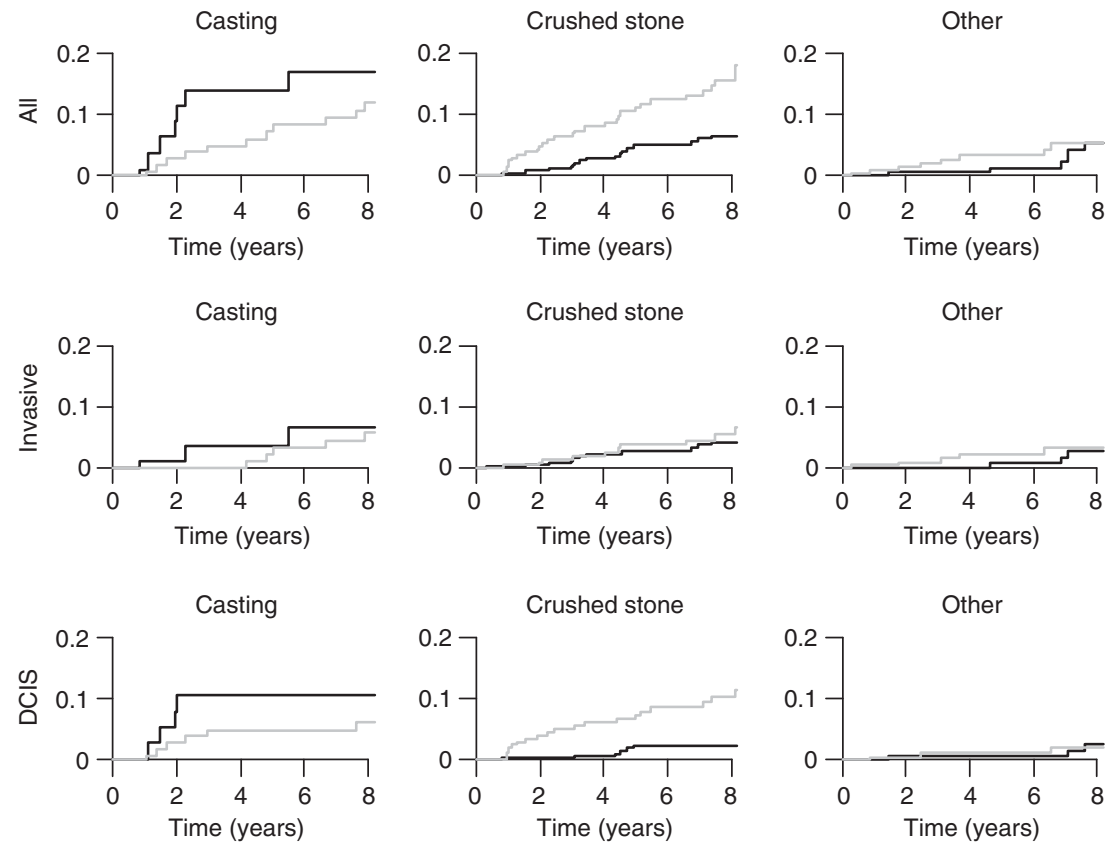

- RT - Control

Figure 3. Cumulative incidence of local recurrences by mammographic pattern in women $\leqslant 56$ years of age at diagnosis; the three upper panels describe all (invasive plus in situ) recurrences in the radiotherapy (RT) arm and the control (Ctrl) arm by type of mammographic pattern of the primary lesion; the three middle panels describe invasive recurrences in the radiotherapy (RT) arm and the control (Ctrl) arm, the lower panels the in situ recurrences in the respective arm of the SweDCIS trial by mammographic pattern of the primary. Women with no calcifications, and those with architectural distortion, galactography findings, and with powdery calcifications are grouped together as 'Other'.
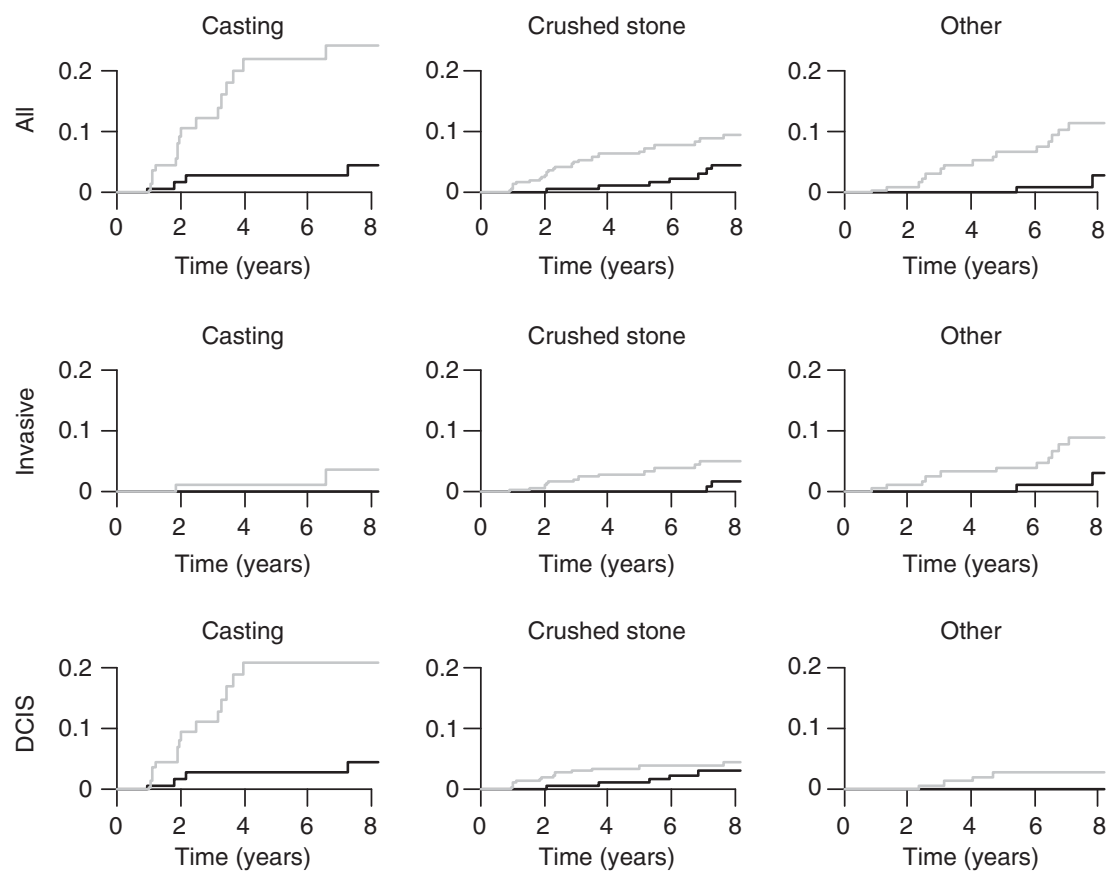

Figure 4. Cumulative incidence of local recurrences by mammographic pattern in women $>56$ years of age at diagnosis; the three upper panels describe all (invasive plus in situ) recurrences in the radiotherapy (RT) arm and the control (Ctrl) arm by type of mammographic pattern of the primary lesion; the three middle panels describe invasive recurrences in the radiotherapy (RT) arm and the control (Ctrl) arm, the lower panels the in situ recurrences in the respective arm of the SweDCIS trial by mammographic pattern of the primary. Women with no calcifications, and those with architectural distortion, galactography findings and powdery calcifications are grouped together as 'Other'.

Several studies (MacMillan et al, 1995; Tabar et al, 2000a and 2004; Malik et al, 2000; Thurfjell et al, 2001; Zunzunegui et al, 2003) have shown that mammography could be helpful at the time of diagnosis to identify women with high-risk invasive disease. However, very few or hardly any data on mammographic patterns in relation to local recurrence were found for women with DCIS 
only. Moreover, comparison between studies is hampered by different classifications of mammographic patterns and microcalcifications that have been used.

A study by Tabar et al, (2000a) proposed that the presence of casting-type calcifications may be a vital prognostic factor in small screen-detected breast cancers. Women with $1-9 \mathrm{~mm}$ and 10-14 mm invasive tumours associated with casting-type calcifications on the mammogram had a 26 -year survival of 72 and $55 \%$, respectively, whereas women with $1-9 \mathrm{~mm}$ and $10-14 \mathrm{~mm}$ invasive cancers without casting type calcifications had a $95 \%$ vs $88 \%$ 26-year disease-specific survival, respectively. Grade 3 DCIS is usually associated with casting-type calcification. Similar conclusions were reached by Thurfjell et al, 2001 when combining the casting type and crushed stone-like calcifications. They investigated 96 women with $1-9 \mathrm{~mm}$ invasive breast cancers with or without calcification, and indicating that casting or pleomorphic calcifications are associated with a significantly higher risk of death, irrespective of nodal status and histological malignancy grade. MacMillan et al (1995) investigated the presence of residual microscopic disease after breast-conservation surgery. They found an association between casting calcifications and prediction of local recurrence. The only independent preoperative mammographic feature was casting-type calcifications as indicated by others (Malik et al, 2000; Zunzunegui et al, 2003).

The fact that crushed stone and particularly casting-type calcifications were associated with in situ recurrence only is of considerable interest. Hypothetically, these patterns often are associated with in situ, multifocal, diffuse in situ growth and can be difficult to excise completely. This hypothesis is corroborated by the rapidly (within four years after surgery) recurring lesions. Also, casting-type calcifications have been associated with multifocality in invasive breast cancer (Kelemen et al, 2012). However, in our study, the RR associated with risk of an in situ recurrence was only marginally altered when disease extent and margin status were taken into account. Another tentative explanation is that this mammographic pattern reveals a susceptibility to develop new in situ lesions. A third possibility is that the lesions associated with casting-type calcifications histologically initially exhibit features of in situ carcinomas even when recurring, but in reality they behave like invasive tumours by ductneogenesis (Tabár et al, 2007). They are thus biologically aggressive and underway to progress to invasive carcinomas. This hypothesis gets some support from the studies of mammographic patterns in invasive cancer cited above. In this study, the histopathological evaluation was not designed with this question in mind and we cannot directly address the ductneogenesis hypothesis.

The indication that age affects responsiveness to radiotherapy in women with casting-type lesions coincides with other findings from this study (Ringberg et al, 2007; Holmberg et al, 2008). First, casting-type calcifications are associated with necrosis and we previously found less effect of radiotherapy in the presence of necrosis (Ringberg et al, 2007). We hypothesised that this was due to less effect of radiotherapy in tissue with low oxygen supply. Second, we found a substantial effect of radiotherapy in terms of absolute risk reduction in older women, but less so in younger women in the last update of SweDCIS (Holmberg et al, 2008). It may be clinically important to study if this age-effect is mainly explained by the difference in responsiveness in the group of women with casting-type calcifications as indicated by the findings in the present study. The cumulative incidence curves for in situ recurrences by randomisation to radiotherapy may imply resistance to radiotherapy in women $\leqslant 56$ years and with casting-type calcifications. As our data come from a randomized trial, the findings related to radiotherapy are not confounded by indication and may well therefore reflect a clinically highly relevant effect. However, we caution that the findings relate to a subgroup analysis with limited statistical precision and should not be taken to guide practice, but as findings to be validated in other cohorts and further understood by biological studies.

In conclusion, crushed stone-like and especially castingtype calcifications are associated with higher risk of in situ recurrence after surgery for in situ breast carcinoma. This high risk is well alleviated by radiotherapy. However, our findings indicate that radiotherapy for these lesions may be less effective in younger women. Both our previous findings on lower responsiveness to radiotherapy in relation to age and these new findings relating to mammographic calcifications indicate that these clinical characteristics together with other factors can help to define groups of women with DCIS where mastectomy with or without reconstruction is a safer option than breast conservation.

\section{ACKNOWLEDGEMENTS}

This study was supported by the Swedish Cancer Society and Västerbotten County, Sweden. Holmberg and Gamso was also supported by Cancer Research UK.

\section{CONFLICT OF INTEREST}

The authors declare no conflict of interest.

\section{REFERENCES}

Emdin SO, Granstrand B, Ringberg A, Sandelin K, Arnesson LG, Nordgren H, Anderson H, Garmo H, Holmberg L, Wallgren A (2006) SweDCIS: Radiotherapy after sector resection for ductal carcinoma in situ of the breast. Results of a randomised trial in a population offered mammography screening. Acta Oncol 45: 536-543.

Goodman DN, Boutross-Tadross O, Jong RA (1995) Mammographic features of pure mucinous carcinoma of the breast with pathological correlation. Can Assoc Radiol J 46: 296-301.

Hermann G, Keller RJ, Drossman S, Caravella BA, Tartter P, Panetta RA, Bleiweiss IJ (2009) Mammographic pattern of microcalcifications in the preoperative diagnosis of comedo ductal carcinoma in situ: histopathologic correlation. Can Assoc Radiol J 50: 235-240.

Hilleren DJ, Andersson IT, Lindholm K, Linnell FS (1991) Invasive lobular carcinoma: mammographic findings in a 10-year experience. Radiology 178: $149-154$.

Holmberg L, Garmo H, Granstrand B, Ringberg A, Arnesson LG, Sandelin K, Karlsson P, Anderson H, Emdin S (2008) Absolute risk reduction of local recurrence after postoperative radiotherapy after sector resection for ductal carcinoma in situ of the breast. J Clin Oncol 26: 1247-1252.

Kalbfleisch JD, Prentice RL (2002) The Statistical Analysis of Failure Time Data. 2nd edn. Wiley: Hoboken, NJ, p 255.

Kelemen G, Farkas V, Debrah J, Ormandi IK, Voros A, Kaizer L, Varqua Z, Lazar G, Kahan Z (2012) The relationship of multifocality and tumor buden with various tumor charactersitcs and survival in early breast cancer. Neoplasma 59: 566-573.

Lenya M (1988) Diagnosis and Differential Diagnosis of Breast Calcifications. Springer-Verlag: Berlin.

MacMillan RD, Purushotham AD, Cordiner C, Dobson H, Mallon E, George WD (1995) Predicting local recurrence by correlating pre-operative mammographic findings with pathological risk factors in patients with breast cancer. Br J Radiol 68: 445-449.

Malik HZ, Wilkinson L, George WD, Purushotham AD (2000) Preoperative mammographic features predict clinicopathological risk factors for the development of local recurrence in breast cancer. Breast 9: 329-333.

Pálka I, Ormándi K, Gaál S, Boda K, Kahán Z (2007) Casting-type calcifications on the mammogram suggest a higher probability of early relapse and death among high-risk breast cancer patients. Acta Oncol 46(8): 1178-1183.

R Development Core Team (2005) A language and environment for statistical computing. R Foundation for Statistical Computing. 
Vienna, Austria. ISBN 3-900051-07-0, URL. Available from http://www. R-project.org.

Ringberg A, Nordgren H, Thorstensson S, Idvall I, Garmo H, Granstrand B, Arnesson LG, Sandelin K, Wallgren A, Anderson H, Emdin S,

Holmberg L (2007) Histopathological risk factors for ipsilateral breast events after breast conserving treatment for ductal carcinoma in situ of the breast-Results from the Swedish randomised trial. Eur J Cancer 43: 291-298.

Self SG, Prentice RL (1988) Asymptotic distribution theory and efficiency results for case-cohort studies. Ann Stat 16: 64-81.

Tabár L, Chen HH, Duffy SW, Yen MF, Chiang CF, Dean PB, Smith RA (2000a) A novel method for prediction of long-term outcome of women with T1a, T1b, and 10-14 mm invasive breast cancers: a prospective study. Lancet 355: 429-433.

Tabár L, Dean PB, Kaufman CS, Duffy SW, Chen HH (2000b) A new era in the diagnosis of breast cancer. Surg Oncol Clin N Am 9: 233-277.

Tabár L, Tony Chen HH, Amy Yen MF, Tot T, Tung TH, Chen LS, Chiu YH, Duffy SW, Smith RA (2004) Mammographic tumor features can predict long-term outcomes reliably in women with 1-14 mm invasive breast carcinoma. Cancer 101: 1745-1759.

Tabár L, Tot T, Dean PB (2005) Breast Cancer: The Art and Science of Early Detection with Mammography. Thieme: Stuttgart.
Tabár L, Tot T, Dean PB (2007) Casting type calcifications: sign of a subtype with deceptive features. Breast Cancer: Early detection with mammography. Thieme: Stuttgart.

Therneau TM, Li H (1998) Computing the Cox model for case cohort designs. Technical Report Number 62, June, Technical Report Series, Section of Biostatistics, Mayo Clinic, Rochester, Minnesota. Available from http:// mayoresearch.mayo.edu/mayo/research/biostat/upload/62.pdf.

Thurfjell E, Thurfjell MG, Lindgren A (2001) Mammographic finding as predictor of survival in 1-9 mm invasive breast cancers. Worse prognosis for cases presenting as calcifications alone. Breast Cancer Res Treat 67: $177-180$.

Wilson TE, Helvie MA, Oberman HA, Joynt LK (1995) Pure and mixed mucinous carcinoma of the breast: pathologic basis for differences in mammographic appearance. Am J Roentgenol 165: 285-289.

Zunzunegui RG, Chung MA, Oruwari J, Golding D, Marchant DJ, Cady B (2003) Casting-type calcifications with invasion and high-grade ductal carcinoma in situ. Arch Surg 138: 537-540.

This work is published under the standard license to publish agreement. After 12 months the work will become freely available and the license terms will switch to a Creative Commons AttributionNonCommercial-Share Alike 3.0 Unported License. 